J. Nonlinear Var. Anal. 5 (2021), No. 2, pp. 299-318

Available online at http://jnva.biemdas.com

https://doi.org/10.23952/jnva.5.2021.2.08

\title{
INFINITELY MANY FAST HOMOCLINIC SOLUTIONS OF DAMPED VIBRATION SYSTEMS WITH SUPERQUADRATIC CONDITIONS OR COMBINED NONLINEARITIES
}

\author{
MOHSEN TIMOUMI \\ Department of Mathematics, Monastir University, Monastir, Tunisia
}

\begin{abstract}
In this paper, we deal with the existence of infinitely many fast homoclinic solutions for damped vibration systems where the nonlinearity is superquadratic or involves a combination of superquadratic and subquadratic terms at infinity and the matrix $L$ is not required to be either uniformly positive definite or coercive.
\end{abstract}

Keywords. Damped vibration systems; Fast homoclinic solutions; Variational methods; Cerami's condition; Combined nonlinearities.

\section{INTRODUCTION}

In the end of the 19th century, Poincare recognized the importance of homoclinic solutions of dynamical systems. It is well known that homoclinic orbits play an important role in analyzing the chaos of dynamical systems. If a system has the transversely intersected homoclinic orbits, then it must be chaotic. If it has the smoothly connected homoclinic orbits, then it cannot stand the perturbation, and its perturbed system probably produce chaotic phenomena. In this paper, we consider the following damped vibration system

$$
\ddot{u}(t)+q(t) \dot{u}(t)-L(t) u(t)+\nabla W(t, u(t))=0, \forall t \in \mathbb{R}
$$

where $q: \mathbb{R} \longrightarrow \mathbb{R}$ is a continuous function such that $Q(t)=\int_{0}^{t} q(s) d s \longrightarrow+\infty$ as $|t| \longrightarrow \infty$, $L \in C\left(\mathbb{R}, \mathbb{R}^{N^{2}}\right)$ is a symmetric matrix-valued function and $W: \mathbb{R} \times \mathbb{R}^{N} \longrightarrow \mathbb{R}$ is a continuous function, differentiable in the second variable with continuous derivative $\frac{\partial W}{\partial x}(t, x)=\nabla W(t, x)$. It is of practical importance and mathematical significance to consider the existence of homoclinic orbits of $(\mathscr{D} \mathscr{V})$ emanating from zero. When $q(t)=0$ for all $t \in \mathbb{R},(\mathscr{D} \mathscr{V})$ is just the following second order Hamiltonian system

$(\mathscr{H} \mathscr{S}) \quad \ddot{u}(t)-L(t) u(t)+\nabla W(t, u(t))=0, \forall t \in \mathbb{R}$,

which is a classical equation that play an important role in practical problems concerning relativistic mechanics, gas dynamics, nuclear physics, fluid mechanics and others. If $L(t)$ and $W(t, x)$ are independent of $t$ or periodic in $t$, the existence and multiplicity of homoclinic solutions for the Hamiltonian system $(\mathscr{H} \mathscr{S})$ have been studied via critical point theory and variational methods; see $[1,2,3,4,5,6]$ and the references cited therein. In this case, the existence

E-mail address: m_timoumi@yahoo.com.

Received February 2, 2020; Accepted March 8, 2021.

(C)2021 Journal of Nonlinear and Variational Analysis 
of homoclinic solutions can be obtained as a limit of periodic solutions of approximating problems. If $L(t)$ and $W(t, x)$ are neither autonomous nor periodic in $t$, the problem is quite different from the periodic case because of the lack of compactness of the Sobolev embedding. In 1991, Rabinowitz and Tanaka [7] first considered this case and obtained the existence of homoclinic solutions for $(\mathscr{H} \mathscr{S})$ when $W$ satisfies the well-known Ambrosetti-Rabinowitz (in short $(\mathscr{A} \mathscr{R})$ ) condition and $L$ satisfies the following coercive condition

$(L) L(t)$ is a positive symmetric matrix for all $t \in \mathbb{R}$ and the smallest eigenvalue of $L(t)$

$$
l(t)=\inf _{|\xi|=1} L(t) \xi \cdot \xi \longrightarrow+\infty \text { as }|t| \longrightarrow \infty
$$

where "." denotes the inner product in $\mathbb{R}^{N}$ and $|$.$| is the induced norm. Indeed, the condition (L)$ can helps us recover the compactness of the Sobolev embedding. Recently, it has been extensively applied in the study of the existence of homoclinic solutions for second order nonperiodic systems. We refer the reader to the references [7]-[19]. In recent years, the research on how to weaken the condition $(L)$ attracted much attention (see, for example, $[20,21,22]$ ). In particular, Jiang and Zhang [20] proved the existence of infinitely many homoclinic solutions for the Hamiltonian system $(\mathscr{H} \mathscr{S})$ when the potential $W$ satisfies some superquadratic conditions at infinity and the matrix-valued function $L$ is neither coercive nor uniformly positive definite. More precisely, $L$ is required to satisfy

$\left(L_{1}\right)$ The smallest eigenvalue $l(t)$ of $L(t)$ is bounded from below;

$\left(L_{0}\right)$ There exists a constant $r_{0}>0$ such that

$$
\lim _{|s| \rightarrow \infty} \operatorname{meas}\left(\{t \in] s-r_{0}, s+r_{0}\left[/ L(t)<M I_{N}\right\}\right)=0, \forall M>0,
$$

where meas denotes the Lebesgue's measure on $\mathbb{R}$. In [18], Wei and Wang obtained the existence of infinitely many homoclinic solutions for the system $(\mathscr{H} \mathscr{S})$ with the case that $W(t, x)=U(t, x)+V(t, x)$, where $U(t, x)$ is subquadratic and $V(t, x)$ is superquadratic by using the following condition

$\left(L_{\gamma}\right)$ The exists a constant $\gamma<0$ such that

$$
l(t)|t|^{\gamma-1} \longrightarrow+\infty \text { as }|t| \longrightarrow+\infty
$$

Compared with the case where $q(t)=0$ for all $t \in \mathbb{R}$, the case where $q(t) \neq 0$ is more difficult and it was only considered by few authors; see, e.g., [23]-[30]. In [29], the author studied the existence of infinitely many fast homoclinic solutions for $(\mathscr{D} \mathscr{V})$ (see Definition 2.1) in the case that $L(t)$ is coercive satisfying the condition $(L)$ and $W(t, x)$ satisfies some superquadratic conditions at infinity. The purpose of this paper is devoted to proving the existence of infinitely many fast homoclinic solutions for system $(\mathscr{D} \mathscr{V})$ when $L(t)$ is not coercive and the potential $W(t, x)$ is either superquadratic at infinity in the second variable and does not need to satisfy the $(\mathscr{A} \mathscr{R})$-condition or involves a combination of subquadratic and superquadratic terms. To the best of our knowledge, it seems that no similar results are obtained in the literature for damped vibration systems. The remainder of this paper is organized as follows. In Section 2, some preliminary results are presented. In Section 3, we deal with the superquadratic conditions. Section 4, the last section, is devoted to the combined nonlinearities. 


\section{PRELIMINARIES}

In order to introduce the concept of fast homoclinic solutions for $(\mathscr{D} \mathscr{V})$ conveniently, we first describe some properties of the weighted Sobolev space $E$ on which the certain variational functional associated with $(\mathscr{D} \mathscr{V})$ is defined and the fast homoclinic solutions of $(\mathscr{D} \mathscr{V})$ are the critical points of such functional. We shall use $L_{Q}^{2}(\mathbb{R})$ to denote the Hilbert space of measurable functions from $\mathbb{R}$ into $\mathbb{R}^{N}$ under the inner product

$$
<u, v>_{L_{Q}^{2}}=\int_{\mathbb{R}} e^{Q(t)} u(t) \cdot v(t) d t
$$

and the induced norm

$$
\|u\|_{L_{Q}^{2}}=\left(\int_{\mathbb{R}} e^{Q(t)}|u(t)|^{2} d t\right)^{\frac{1}{2}} .
$$

Similarly, $L_{Q}^{s}(\mathbb{R})(1 \leq s<\infty)$ denotes the Banach space of functions on $\mathbb{R}$ with values in $\mathbb{R}^{N}$ under the norm

$$
\|u\|_{L_{Q}^{s}}=\left(\int_{\mathbb{R}} e^{Q(t)}|u(t)|^{s} d t\right)^{\frac{1}{s}}
$$

and $L_{Q}^{\infty}(\mathbb{R})$ denotes the Banach space of functions on $\mathbb{R}$ with values in $\mathbb{R}^{N}$ under the norm

$$
\|u\|_{L_{Q}^{\infty}}=\operatorname{essup}\left\{e^{\frac{Q(t)}{2}}|u(t)| / t \in \mathbb{R}\right\} .
$$

In this section, we assume that $L$ satisfies the following condition instead of the condition $\left(L_{1}\right)$ $\left(L_{1}^{\prime}\right)$ There exists a positive constant $l_{0}$ such that

$$
l(t)=\inf _{|\xi|=1} L(t) \xi \cdot \xi \geq l_{0}, \forall t \in \mathbb{R}
$$

and we introduce the Hilbert space

$$
E=\left\{u \in H_{Q}^{1}(\mathbb{R}) / \int_{\mathbb{R}} e^{Q(t)} L(t) u(t) \cdot u(t) d t<\infty\right\}
$$

equipped with the following inner product

$$
<u, v>=\int_{\mathbb{R}} e^{Q(t)}(\dot{u}(t) \cdot \dot{v}(t)+L(t) u(t) \cdot v(t)) d t
$$

and the induced norm $\|u\|=<u, u>^{\frac{1}{2}}$. Here, $H_{Q}^{1}(\mathbb{R})$ denotes the Sobolev space

$$
H_{Q}^{1}(\mathbb{R})=\left\{u \in L_{Q}^{2}(\mathbb{R}) / \dot{u} \in L_{Q}^{2}(\mathbb{R})\right\} .
$$

Evidently, $E$ is continuously embedded into $L_{Q}^{s}(\mathbb{R})$ for $2 \leq s \leq \infty$, i.e., for all $2 \leq s \leq \infty$. There exists a constant $\eta_{s}>0$ such that

$$
\|u\|_{L_{Q}^{s}} \leq \eta_{s}\|u\|, \forall u \in E .
$$

Definition 2.1. A solution $u$ of $(\mathscr{D} \mathscr{V})$ is called a fast homoclinic orbit if $u \in E$.

Lemma 2.1. Assume that $\left(L_{1}^{\prime}\right)$ and $\left(L_{Q}\right)$ are satisfied. Then $E$ is compactly embedded in $L_{Q}^{s}(\mathbb{R})$ for all $2 \leq s<\infty$. 
Proof. Let $\left(u_{n}\right) \subset E$ be a bounded sequence such that $u_{n} \rightarrow u$ in $E$. We shall show that $u_{n} \longrightarrow u$ in $L_{Q}^{S}(\mathbb{R})$ for all $2 \leq s<\infty$.

-Step 1. Consider the case $s=2$. Suppose, without loss of generality, that $u_{n} \rightarrow 0$ in $E$. For any $s \in \mathbb{R}$, we denote $I_{r_{0}}(s)$ the open interval in $\mathbb{R}$ centered at $s$ with radius $r_{0}$, i.e., $I_{r_{0}}(s)=$ ]$s-r_{0}, s+r_{0}$ [, where $r_{0}$ is the constant given in $\left(L_{Q}\right)$. Let $\left(s_{i}\right)_{i \in \mathbb{N}} \subset \mathbb{R}$ be a sequence of points such that $\mathbb{R}=\cup_{i=1}^{\infty} I_{r_{0}}\left(s_{i}\right)$ and each $t \in \mathbb{R}$ is contained in at most two such intervals. For any $r>0$ and $b>0$, let

$$
\begin{aligned}
& C(r, b)=\{t \in \mathbb{R} \backslash]-r, r\left[/ L(t) \geq b I_{N}\right\}, \\
& D(r, b)=\{t \in \mathbb{R} \backslash]-r, r\left[/ L(t)<b I_{N}\right\} .
\end{aligned}
$$

Choosing $b_{\varepsilon}>\frac{4}{\varepsilon} \sup _{n \in \mathbb{N}}\left\|u_{n}\right\|^{2}$, we have

$$
\begin{aligned}
\int_{C\left(r, b_{\varepsilon}\right)} e^{Q(t)}\left|u_{n}\right|^{2} d t & \leq \frac{1}{b_{\varepsilon}} \int_{C\left(r, b_{\varepsilon}\right)} e^{Q(t)} L(t) u_{n}(t) \cdot u_{n}(t) d t \\
& \leq \frac{1}{b_{\varepsilon}} \int_{\mathbb{R}} e^{Q(t)} L(t) u_{n}(t) \cdot u_{n}(t) d t \\
& \leq \frac{1}{b_{\varepsilon}}\left\|u_{n}\right\|^{2}<\frac{\varepsilon}{4}
\end{aligned}
$$

Now, we have

$$
\begin{aligned}
\int_{D\left(r, b_{\varepsilon}\right)} e^{Q(t)}\left|u_{n}\right|^{2} d t & \leq \sum_{i=1}^{\infty} \int_{D\left(r, b_{\varepsilon}\right) \cap I_{r_{0}}\left(s_{i}\right)} e^{Q(t)}\left|u_{n}\right|^{2} d t \\
& \leq \sum_{i=1}^{\infty}\left(\int_{D\left(r, b_{\varepsilon}\right) \cap I_{r_{0}}\left(s_{i}\right)} e^{Q(t)}\left|u_{n}\right|^{4} d t\right)^{\frac{1}{2}}\left[\operatorname{meas}_{Q}\left(D\left(r, b_{\varepsilon}\right) \cap I_{r_{0}}\left(s_{i}\right)\right)\right]^{\frac{1}{2}} \\
& \leq a_{r} \sum_{i=1}^{\infty}\left(\int_{I_{r_{0}}\left(s_{i}\right)} e^{Q(t)}\left|u_{n}\right|^{4} d t\right)^{\frac{1}{2}}
\end{aligned}
$$

where $a_{r}=\sup _{i \in \mathbb{N}}\left[\operatorname{meas}_{Q}\left(D\left(r, b_{\varepsilon}\right) \cap I_{r_{0}}\left(s_{i}\right)\right)\right]^{\frac{1}{2}}$. From (2.1), we have

$$
\begin{aligned}
\left(\int_{I_{r_{0}}\left(s_{i}\right)} e^{Q(t)}\left|u_{n}\right|^{4} d t\right)^{\frac{1}{4}} & =\left(\int_{\mathbb{R}} e^{Q(t)}\left|\chi_{\mid I_{r_{0}}\left(s_{i}\right)} u_{n}\right|^{4} d t\right)^{\frac{1}{4}} \\
& \leq \eta_{4}\left\|\chi_{\mid I_{r_{0}}\left(s_{i}\right)} u_{n}\right\| \leq \eta_{4}\left\|u_{n}\right\| .
\end{aligned}
$$

Hence

$$
\begin{aligned}
\int_{D\left(r, b_{\varepsilon}\right)} e^{Q(t)}\left|u_{n}\right|^{2} d t & \leq \eta_{4}^{2} a_{r} \sum_{i=1}^{\infty}\left\|\chi_{\mid I_{r_{0}}\left(s_{i}\right)} u_{n}\right\|^{2} \\
& =\eta_{4}^{2} a_{r} \sum_{i=1}^{\infty} \int_{I_{r_{0}}\left(s_{i}\right)} e^{Q(t)}\left[\left|\dot{u}_{n}\right|^{2}+L(t) u_{n}(t) \cdot u_{n}(t)\right] d t \\
& \leq 2 \eta_{4}^{2} a_{r} \sup _{n \in \mathbb{N}}\left\|u_{n}\right\|^{2} .
\end{aligned}
$$

By an easy computation, we show that $a_{r} \longrightarrow 0$ as $r \longrightarrow \infty$. Therefore there exists $r_{\varepsilon}>0$ such that

$$
\int_{D\left(r_{\varepsilon}, b_{\varepsilon}\right)} e^{Q(t)}\left|u_{n}\right|^{2} d t<\frac{\varepsilon}{4}
$$


which together with (2.2) implies

$$
\int_{\mathbb{R} \backslash]-r_{\varepsilon}, r_{\varepsilon}[} e^{Q(t)}\left|u_{n}\right|^{2} d t=\int_{C\left(r_{\varepsilon}, b_{\varepsilon}\right)} e^{Q(t)}\left|u_{n}\right|^{2} d t+\int_{D\left(r_{\varepsilon}, b_{\varepsilon}\right)} e^{Q(t)}\left|u_{n}\right|^{2} d t<\frac{\varepsilon}{2} .
$$

By Sobolev's theorem, $u_{k} \longrightarrow 0$ uniformly on $\left[-r_{\varepsilon}, r_{\varepsilon}\right]$. Then there exists $k_{0}>0$ such that

$$
\int_{\left[-r_{\varepsilon}, r_{\varepsilon}\right]}\left|u_{k}\right|^{2} d t<\frac{\varepsilon}{2}, \forall k \geq k_{0}
$$

Combining (2.3) with (2.4), and using the arbitrary of $\varepsilon$, we can obtain that $u_{k} \longrightarrow 0$ in $L_{Q}^{2}(\mathbb{R})$.

-Step 2. Case: $2<s<\infty$. From [20, Lemma 2.3], for any $v \in H^{1}(\mathbb{R})$, we have

$$
\|v\|_{L^{\infty}} \leq \frac{\sqrt{2}}{2}\left[\int_{\mathbb{R}}\left(|\dot{v}|^{2}+|v|^{2}\right) d t\right]^{\frac{1}{2}}
$$

Let $u \in E$ and set $v(t)=e^{\frac{Q(t)}{2}} u(t)$. By $\left(L_{1}^{\prime}\right)$, one has

$$
\begin{aligned}
\int_{\mathbb{R}}\left(|\dot{v}|^{2}+|v|^{2}\right) d t & =\int_{\mathbb{R}} e^{Q(t)}\left(\left|\dot{u}+\frac{q}{2} u\right|^{2}+|u|^{2}\right) d t \\
& \leq \int_{\mathbb{R}} e^{Q(t)}\left[2|\dot{u}|^{2}+\left(1+\frac{\|q\|_{L^{\infty}}^{2}}{2}\right)|u|^{2}\right] d t \\
& \leq \int_{\mathbb{R}} e^{Q(t)}\left[2|\dot{u}|^{2}+l_{0}^{-1}\left(1+\frac{\|q\|_{L^{\infty}}^{2}}{2}\right) L(t) u(t) \cdot u(t)\right] d t \\
& \leq c^{2}(q)\|u\|^{2}
\end{aligned}
$$

where $c^{2}(q)=\max \left\{2, l_{0}^{-1}\left(1+\frac{\|q\|_{L^{\infty}}^{2}}{2}\right)\right\}$. Combining (2.5) and (2.6) yields

$$
\|u\|_{L_{Q}^{\infty}} \leq \frac{\sqrt{2}}{2} c(q)\|u\|
$$

Hence

$$
\begin{aligned}
\left(\int_{\mathbb{R}} e^{Q(t)}|u|^{s} d t\right)^{\frac{1}{s}} & =\left(\int_{\mathbb{R}} e^{Q(t)}|u|^{2}|u|^{s-2} d t\right)^{\frac{1}{s}} \\
& \leq\|u\|_{L^{\infty}}^{\frac{s-2}{2}}\|u\|_{L_{Q}^{2}}^{\frac{2}{s}} \\
& \left.\leq\left(\frac{1}{m_{0}}\right)^{\frac{s-2}{2 s}}\|u\|_{L_{Q}^{\frac{s-2}{s}}}^{s}\|u\|_{L_{Q}^{\frac{2}{s}}}^{\frac{2}{\sqrt{2}}} c^{2}(q)\right)^{\frac{s-2}{2 s}}\|u\|^{\frac{s-2}{s}}\|u\|_{L_{Q}^{2}}^{\frac{2}{s}}, \\
& \leq\left(\frac{m_{0}}{2}\right.
\end{aligned}
$$

where $m_{0}=\min _{t \in \mathbb{R}} e^{Q(t)}$. By virtue of Step 1 and (2.7), we obtain $u_{n} \longrightarrow 0$ in $L_{Q}^{S}(\mathbb{R})$. The proof of Lemma 2.1 is completed.

To study the critical points of the variational functional associated with $(\mathscr{D} \mathscr{V})$, we need to recall the following critical point theorems. 
Definition 2.2. Let $E$ be a Banach space with the norm $\|$. $\|$. One says that $\psi \in C^{1}(E, \mathbb{R})$ satisfies the

a) $(P S)_{c}$-condition, $c \in \mathbb{R}$ if any sequence $\left(u_{n}\right) \subset E$ satisfying

$$
\psi\left(u_{n}\right) \longrightarrow c \text { and } \psi^{\prime}\left(u_{n}\right) \longrightarrow 0 \text { as } n \longrightarrow \infty
$$

possesses a convergent subsequence,

b) $(C)_{c}$-condition, $c \in \mathbb{R}$ if any sequence $\left(u_{n}\right) \subset E$ satisfying

$$
\psi\left(u_{n}\right) \longrightarrow c \text { and }\left\|\psi^{\prime}\left(u_{n}\right)\right\|\left(1+\left\|u_{n}\right\|\right) \longrightarrow 0 \text { as } n \longrightarrow \infty
$$

possesses a convergent subsequence.

Lemma 2.2. (Symmetric Mountain Pass Theorem) [31] Let E be an infinite dimensional Banach space, and $E=Y \oplus Z$, where $Y$ is finite dimensional space. Suppose that $\psi \in C^{1}(E, \mathbb{R})$ satisfies the Palais-Smale condition and

$$
\psi(0)=0, \psi(-u)=\psi(u), \forall u \in E
$$

(2) There exist constants $\rho, \alpha>0$ such that $\psi \mid \partial B_{\rho} \cap Z \geq \alpha$;

(3) For any finite dimensional subspace $\widetilde{E} \subset E$, there is $R=R(\widetilde{E})>0$ such that $\psi(u) \leq 0$ on $\widetilde{E} \backslash B_{R}$, where $B_{R}=\{u \in E /\|u\|<R\}$.

Then $\psi$ possesses an unbounded sequence of critical values.

Remark 2.1. As shown in [32], a deformation lemma can be proved with $(C)_{c}$-condition replacing the $(P S)_{c}$-condition, and it turns out that Lemma 2.2 still holds true with the $(C)_{c}$-condition instead of the $(P S)_{c}$-condition.

Now, let $E$ be a Banach space with the norm $\|$.$\| and E=\overline{\oplus_{j \in \mathbb{N}} X_{j}}$, where $X_{j}$ is a finite dimensional subspace of $E$. For each $k \in \mathbb{N}$, let $Y_{k}=\oplus_{j=0}^{k} X_{j}, Z_{k}=\overline{\oplus_{j=k}^{\infty} X_{j}}$. The functional $\psi \in C^{1}(E, \mathbb{R})$ is said to satisfy the $(P S)^{*}$ condition if for any sequence $\left(u_{j}\right)$ for which $\left(\psi\left(u_{j}\right)\right)$ is bounded, $u_{j} \in Y_{k_{j}}$ for some $k_{j}$ with $k_{j} \longrightarrow \infty$ and $\left(\psi_{\mid Y_{k_{j}}}\right)^{\prime}\left(u_{j}\right) \longrightarrow 0$ as $j \longrightarrow \infty$, has a subsequence converging to a critical point of $\psi$.

Lemma 2.3. (Dual Fountain Theorem) [33] Suppose that the functional $\psi \in C^{1}(E, \mathbb{R})$ is even and satisfies the $(P S)^{*}$ condition. Assume that, for each sufficiently large integer $k$, there exist $0<r_{k}<\rho_{k}$ such that

$$
\begin{gathered}
a_{k}=\inf _{u \in Z_{k},\|u\|=\rho_{k}} \psi(u) \geq 0 ; \\
b_{k}=\max _{u \in Y_{k},\|u\|=r_{k}} \psi(u)<0 ; \\
d_{k}=\inf _{u \in Z_{k},\|u\| \leq \rho_{k}} \psi(u) \longrightarrow 0 \text { as } k \longrightarrow \infty .
\end{gathered}
$$

Hence $\psi$ has a sequence of negative critical values converging to zero. 


\section{SupERQuAdRATIC CONDITIONS}

In this section, we deal with the superquadratic case and we introduce the following hypotheses on the matrix $L(t)$ and the potential $W(t, x)$.

$\left(L_{Q}\right)$ There exists a constant $r_{0}>0$ such that

$$
\lim _{|s| \rightarrow \infty} \operatorname{meas}_{Q}\left(\{t \in] s-r_{0}, s+r_{0}\left[/ L(t)<M I_{N}\right\}\right)=0, \forall M>0,
$$

where meas $_{Q}$ denotes the Lebesgue's measure on $\mathbb{R}$ with density $e^{Q(t)}$, i.e., meas ${ }_{Q}(A)=\int_{A} e^{Q(t)} d t$; $\left(W_{1}\right)$ There exist constants $a, b>0$ and $p>2$ such that

$$
|\nabla W(t, x)| \leq a|x|+b|x|^{p-1}, \forall(t, x) \in \mathbb{R} \times \mathbb{R}^{N} ;
$$

$$
\lim _{|x| \rightarrow \infty} \frac{W(t, x)}{|x|^{2}}=+\infty \text { uniformly in } t \in \mathbb{R}
$$

and $W(t, x)$ is bounded from below;

$$
\begin{gathered}
W(t,-x)=W(t, x), \forall(t, x) \in \mathbb{R} \times \mathbb{R}^{N} ; \\
\nabla W(t, x) \cdot x-2 W(t, x) \geq 0, \forall(t, x) \in \mathbb{R} \times \mathbb{R}^{N}
\end{gathered}
$$

and there exist constants $c_{0}>0, r_{0} \geq 0$ and $\sigma>1$ such that

$$
|W(t, x)|^{\sigma} \leq c_{0}|x|^{2 \sigma}[\nabla W(t, x) \cdot x-2 W(t, x)], \forall t \in \mathbb{R},|x| \geq r_{0} ;
$$

$\left(W_{4}^{\prime}\right)$ There exist constants $\mu>2$ and $\gamma>0$ such that

$$
\mu W(t, x) \leq \nabla W(t, x) \cdot x+\gamma|x|^{2}, \forall(t, x) \in \mathbb{R} \times \mathbb{R}^{N} .
$$

Our first main results read as follows.

Theorem 3.1. Assume that $\left(L_{1}\right),\left(L_{Q}\right)$ and $\left(W_{1}\right)-\left(W_{4}\right)$ hold. Then $(\mathscr{D} \mathscr{V})$ has infinitely many nontrivial fast homoclinic solutions.

Theorem 3.2. Assume that $\left(L_{1}\right),\left(L_{Q}\right),\left(W_{1}\right)-\left(W_{3}\right)$ and $\left(W_{4}^{\prime}\right)$ hold. Then $(\mathscr{D} \mathscr{V})$ has infinitely many nontrivial fast homoclinic solutions.

Remark 3.1. It is easy to see that conditions $\left(L_{1}\right),\left(L_{Q}\right)$ are weaker than the coercivity condition $\left(L_{\gamma}\right)$. For example

$$
L(t)=\left(|t| \sin ^{2} t-1\right) I_{N}
$$

satisfies $\left(L_{1}\right),\left(L_{Q}\right)$ but is not coercive.

Besides, the usual condition $(\mathscr{A} \mathscr{R})$ is not required in our results. In fact, let

$$
W(t, x)=a(t)\left[\left(4|x|^{2}-1\right) \ln \left(\frac{1}{2}+|x|\right)-2\left(\frac{1}{2}+|x|\right)^{2}+4|x|+\frac{1}{2}-\ln 2\right],
$$

where $a \in C(\mathbb{R}, \mathbb{R})$ is such that $0<\inf _{t \in \mathbb{R}} a(t) \leq \sup _{t \in \mathbb{R}} a(t)<+\infty$. It is clear that $W(t, x)$ satisfies $\left(W_{1}\right)-\left(W_{3}\right)$. It remains to verify $\left(W_{4}\right)$. An easy computation shows that

$$
\nabla W(t, x) \cdot x-2 W(t, x)=a(t)\left[\left(4|x|^{2}-1\right) \frac{2|x|}{2|x|+1}-2|x|+2 \ln \left(\frac{1}{2}+|x|\right)+2 \ln 2\right] .
$$


It is easy to see that $\nabla W(t, x) \cdot x-2 W(t, x) \geq 0$ for all $(t, x) \in \mathbb{R} \times \mathbb{R}^{N}$. Moreover, for all $\sigma>1$, we have

$$
\left(\frac{W(t, x)}{|x|^{2}}\right)^{\sigma}[\nabla W(t, x) \cdot x-2 W(t, x)]^{-1} \cong(4 a(t))^{\sigma-1} \frac{\left(\ln \left(\frac{1}{2}+|x|\right)\right)^{\sigma}}{|x|^{2}},
$$

which converges to 0 as $|x| \longrightarrow \infty$, uniformly in $t \in \mathbb{R}$. Hence there exist two positive constants $r_{0}, c_{0}$ such that

$$
\left(\frac{W(t, x)}{|x|^{2}}\right)^{\sigma} \leq c_{0}[\nabla W(t, x) \cdot x-2 W(t, x)], \forall t \in \mathbb{R},|x| \geq r_{0} .
$$

Therefore $\left(W_{4}\right)$ holds. Meanwhile it is easy to check that the $(\mathscr{A} \mathscr{R})$ condition is not satisfied. By Theorem 3.1, the corresponding damped vibration system $(\mathscr{D} \mathscr{V})$ possesses infinitely many nontrivial fast homoclinic solutions.

From $\left(L_{1}\right)$, we know that there exists a positive constant $l_{0}$ such that $\inf _{t \in \mathbb{R}} \inf _{|\xi|=1} L(t) \xi \cdot \xi+$ $2 l_{0}>l_{0}$. Let $\bar{L}(t)=L(t)+2 l_{0} I_{N}$ and $\bar{W}(t, x)=W(t, x)+l_{0}|x|^{2}$. Consider the following damped vibration system

$$
\ddot{u}(t)+q(t) \dot{u}(t)-\bar{L}(t) u(t)+\nabla \bar{W}(t, u(t))=0, \forall t \in \mathbb{R} .
$$

Then $(\overline{\mathscr{D} \mathscr{V}})$ and $(\mathscr{D} \mathscr{V})$ are equivalent and it is easy to check that the hypotheses $\left(W_{1}\right)-\left(W_{4}\right)$ and $\left(W_{4}^{\prime}\right)$ still hold for $\bar{W}$ provided that those hold for $W$, and $\bar{L}$ satisfies the conditions $\left(L_{1}^{\prime}\right)$, $\left(L_{Q}\right)$. Hence, in what follows, we always assume without loss of generality that $L$ satisfies $\left(L_{1}^{\prime}\right)$ instead of $\left(L_{1}\right)$.

Consider the functional $\psi$ associated to the system $(\mathscr{D} \mathscr{V})$

$$
\begin{aligned}
\psi(u) & =\frac{1}{2} \int_{\mathbb{R}} e^{Q(t)}\left[|\dot{u}(t)|^{2}+L(t) u(t) \cdot u(t)\right] d t-\int_{\mathbb{R}} e^{Q(t)} W(t, u) d t \\
& =\frac{1}{2}\|u\|^{2}-\int_{\mathbb{R}} e^{Q(t)} W(t, u) d t
\end{aligned}
$$

defined on the space $E$ introduced in Section 2. It is well-known that $\psi \in C^{1}(E, \mathbb{R})$ and critical points of $\psi$ are fast homoclinic solutions of $(\mathscr{D} \mathscr{V})$. Letting $\left(e_{j}\right)_{j \in \mathbb{N}}$ be an orthonormal basis of $E$, we set

$$
Y_{m}=\operatorname{span}\left\{e_{1}, \ldots, e_{m}\right\}, Z_{m}=\overline{\operatorname{span}\left\{e_{m+1}, \ldots\right\}}, m \in \mathbb{N} .
$$

Then $E=Y_{m} \oplus Z_{m}$.

Lemma 3.1. Assume that $\left(L_{1}^{\prime}\right),\left(L_{Q}\right)$ and $\left(W_{1}\right)$ are satisfied. Then there exist positive constants $m_{0}, \alpha, \rho$ such that

$$
\psi_{\mid \partial B_{\rho} \cap Z_{m_{0}} \geq \alpha}
$$

Proof. From $\left(W_{1}\right)$, we have

$$
|W(t, x)| \leq \frac{a}{2}|x|^{2}+\frac{b}{p}|x|^{p}, \forall(t, x) \in \mathbb{R} \times \mathbb{R}^{N} .
$$

For any $m \in \mathbb{N}$, let

$$
l_{2}(m)=\sup _{u \in Z_{m} \backslash\{0\}} \frac{\|u\|_{L_{Q}^{2}}}{\|u\|} \text { and } l_{p}(m)=\sup _{u \in Z_{m} \backslash\{0\}} \frac{\|u\|_{L_{Q}^{p}}}{\|u\|} .
$$


It is clear that $l_{2}(m+1) \leq l_{2}(m)$. So, $l_{2}(m) \longrightarrow l \geq 0$ as $m \longrightarrow \infty$. For any $m \in \mathbb{N}$, there exists $u_{m} \in Z_{m}$ such that $\left\|u_{m}\right\|=1$ and $\left\|u_{m}\right\|_{L_{Q}^{2}} \geq \frac{1}{2} l_{2}(m)$. By the definition of $Z_{m}$, and $u_{m} \rightarrow 0$ in $E$, we have that $u_{m} \longrightarrow 0$ in $L_{Q}^{2}(\mathbb{R})$. Hence, $l=0$, that is, $l_{2}(m) \longrightarrow 0$ as $m \longrightarrow \infty$. Similarly, $l_{p}(m) \longrightarrow 0$ as $m \longrightarrow \infty$. Therefore, we can choose a larger integer $m_{0}$ such that

$$
\|u\|_{L_{Q}^{2}}^{2} \leq \frac{1}{2 a}\|u\|^{2},\|u\|_{L_{Q}^{p}}^{p} \leq \frac{p}{4 b}\|u\|^{p}, \forall u \in Z_{m_{0}}
$$

which together with (3.1) implies that

$$
\begin{aligned}
\psi(u) & =\frac{1}{2}\|u\|^{2}-\int_{\mathbb{R}} e^{Q(t)} W(t, u) d t \\
& \geq \frac{1}{2}\|u\|^{2}-\int_{\mathbb{R}} e^{Q(t)}\left(\frac{a}{2}|u|^{2}+\frac{b}{p}|u|^{p}\right) d t \\
& \geq \frac{1}{2}\|u\|^{2}-\frac{a}{2}\|u\|_{L_{Q}^{2}}^{2}-\frac{b}{p}\|u\|_{L_{Q}^{p}}^{p} \\
& \geq \frac{1}{4}\left(\|u\|^{2}-\|u\|^{p}\right) \\
& =\frac{2^{p-2}-1}{2^{p+2}}=\alpha, \forall u \in Z_{m_{0}},\|u\|=\frac{1}{2}=\rho,
\end{aligned}
$$

which finish the proof of Lemma 3.1.

To apply Lemma 2.3, we will take $E=Y \oplus Z$ with $Y=Y_{m_{0}}$ and $Z=Z_{m_{0}}$, where $m_{0}$ is introduced in Lemma 3.1.

Lemma 3.2. Assume that $\left(L_{1}^{\prime}\right),\left(L_{Q}\right),\left(W_{1}\right)$ and $\left(W_{2}\right)$ are satisfied. Then, for any finite dimensional subspace $\tilde{E} \subset E$, there is a constant $R=R(\tilde{E})>0$ such that $\psi(u) \leq 0, \forall u \in \tilde{E},\|u\| \geq R$.

Proof. We only need to prove

$$
\psi(u) \longrightarrow-\infty \text { as }\|u\| \longrightarrow \infty, u \in \tilde{E} .
$$

Assume by contradiction that there exists a sequence $\left(u_{n}\right) \subset \tilde{E}$ with $\left\|u_{n}\right\| \longrightarrow \infty$ as $n \longrightarrow \infty$ and $\psi\left(u_{n}\right) \geq-M$ for some constant $M>0, \forall n \in \mathbb{N}$. Letting $v_{n}=\frac{u_{n}}{\left\|u_{n}\right\|}$, one has $\left\|v_{n}\right\|=1$. Going to a subsequence if necessary, we can assume that $v_{n} \rightarrow v$ in $E$. Since $\tilde{E}$ is finite dimensional, then $v_{n} \longrightarrow v$ in $E$. Thus $\|v\|=1$. Let

$$
\Lambda_{n}(c, d)=\left\{t \in \mathbb{R} / c \leq\left|u_{n}(t)\right|<d\right\}, 0 \leq c<d
$$

and

$$
\Lambda=\{t \in \mathbb{R} / v(t) \neq 0\}
$$


For any $t \in \Lambda$, we have $\lim _{n \longrightarrow \infty}\left|u_{n}(t)\right|=\lim _{n \longrightarrow \infty}\left\|u_{n}\right\|\left|v_{n}(t)\right|=+\infty$. Hence $\Lambda \subset \Lambda_{n}(r, \infty)$ for $n$ large enough. Property (3.1), Lemma 2.2, assumption $\left(W_{2}\right)$ and Fatou's lemma imply

$$
\begin{aligned}
0 & =\lim _{n \longrightarrow \infty} \frac{-M}{\left\|u_{n}\right\|^{2}} \leq \lim _{n \rightarrow \infty} \frac{\psi\left(u_{n}\right)}{\left\|u_{n}\right\|^{2}}=\lim _{n \longrightarrow \infty}\left[\frac{1}{2}-\int_{\mathbb{R}} e^{Q(t)} \frac{W\left(t, u_{n}\right)}{\left|u_{n}\right|^{2}}\left|v_{n}\right|^{2} d t\right] \\
& =\lim _{n \longrightarrow \infty}\left[\frac{1}{2}-\int_{\Lambda_{n}(0, r)} e^{Q(t)} \frac{W\left(t, u_{n}\right)}{\left|u_{n}\right|^{2}}\left|v_{n}\right|^{2} d t-\int_{\Lambda_{n}(r, \infty)} e^{Q(t)} \frac{W\left(t, u_{n}\right)}{\left|u_{n}\right|^{2}}\left|v_{n}\right|^{2} d t\right] \\
& \leq \limsup _{n \longrightarrow \infty}\left[\frac{1}{2}+\left(\frac{a}{2}+\frac{b}{p} r^{p-2}\right) \int_{\mathbb{R}} e^{Q(t)}\left|v_{n}\right|^{2} d t-\int_{\Lambda_{n}(r, \infty)} e^{Q(t)} \frac{W\left(t, u_{n}\right)}{\left|u_{n}\right|^{2}}\left|v_{n}\right|^{2} d t\right] \\
& \leq \frac{1}{2}+\left(\frac{a}{2}+\frac{b}{p} r^{p-2}\right) \eta_{2}^{2}-\liminf _{n \longrightarrow \infty} \int_{\mathbb{R}} e^{Q(t)} \frac{W\left(t, u_{n}\right)}{\left|u_{n}\right|^{2}} \chi_{\Lambda_{n}(r, \infty)}\left|v_{n}\right|^{2} d t \\
& \leq \frac{1}{2}+\left(\frac{a}{2}+\frac{b}{p} r^{p-2}\right) \eta_{2}^{2}-\int_{\mathbb{R}} \liminf _{n \longrightarrow \infty} e^{Q(t)} \frac{W\left(t, u_{n}\right)}{\left|u_{n}\right|^{2}} \chi_{\Lambda_{n}(r, \infty)}\left|v_{n}\right|^{2} d t \\
& =-\infty .
\end{aligned}
$$

It is a contradiction. Hence (3.2) is satisfied and the proof of Lemma 3.2 is finished.

3.1. Proof of Theorem 3.1. By $\left(W_{3}\right)$ and Lemmas 3.1, 3.2, $\psi$ satisfies the conditions (1), (2) and (3) of Lemma 2.2. It remains to prove the Cerami's condition.

Lemma 3.3. Assume that $\left(L_{1}^{\prime}\right),\left(L_{Q}\right),\left(W_{1}\right),\left(W_{2}\right)$ and $\left(W_{4}\right)$ are satisfied. Then $\psi$ satisfies the $(C)_{c}$-condition for any level $c>0$.

Proof. Let $c$ be a positive real and $\left(u_{n}\right) \subset E$ be a $(C)_{c}$-sequence, that is,

$$
\psi\left(u_{n}\right) \longrightarrow c \text { and }\left\|\psi^{\prime}\left(u_{n}\right)\right\|\left(1+\left\|u_{n}\right\|\right) \longrightarrow 0 \text { as } n \longrightarrow \infty .
$$

Assume by contradiction that $\left(u_{n}\right)$ is not bounded, then up to a subsequence, we can assume that $\left\|u_{n}\right\| \longrightarrow \infty$ as $n \longrightarrow \infty$. Let $v_{n}=\frac{u_{n}}{\left\|u_{n}\right\|}$. Then, $\left\|v_{n}\right\|=1$. Taking a subsequence if necessary, then $v_{n} \rightarrow v$ in $E$ and Lemma 2.1 implies that $v_{n} \longrightarrow v$ in $L_{Q}^{q}(\mathbb{R})$ for $q=2, p, \frac{2 \sigma}{\sigma-1}$ and $v_{n} \longrightarrow v$ a.e. on $\mathbb{R}$. If $v \neq 0$, then

$$
0=\lim _{n \rightarrow \infty} \frac{\psi\left(u_{n}\right)}{\left\|u_{n}\right\|^{2}}=\lim _{n \rightarrow \infty}\left[\frac{1}{2}-\int_{\mathbb{R}} e^{Q(t)} \frac{W\left(t, u_{n}\right)}{\left|u_{n}\right|^{2}}\left|v_{n}\right|^{2} d t\right] \leq-\infty,
$$

which is a contradiction. So $\left(u_{n}\right)$ is bounded. If $v=0$, then $v_{n} \longrightarrow 0$ in $L_{Q}^{q}(\mathbb{R})$ for $q=2, p, 2 \sigma^{\prime}=$ $\frac{2 \sigma}{\sigma-1}$. On one hand, since $\psi\left(u_{n}\right) \longrightarrow c$ and $\left\|u_{n}\right\| \longrightarrow \infty$, then it is easy to see that

$$
\limsup _{n \rightarrow \infty} \int_{\mathbb{R}} e^{Q(t)} \frac{W\left(t, u_{n}\right)}{\left|u_{n}\right|^{2}}\left|v_{n}\right|^{2} d t \geq \frac{1}{2} .
$$

On the other hand, (3.1) implies

$$
\begin{aligned}
\int_{\Lambda_{n}(0, r)} e^{Q(t)} \frac{W\left(t, u_{n}\right)}{\left|u_{n}\right|^{2}}\left|v_{n}\right|^{2} d t & \leq\left(\frac{a}{2}+\frac{b}{p} r^{p-2}\right) \int_{\Lambda_{n}(0, r)} e^{Q(t)}\left|v_{n}\right|^{2} d t \\
& \leq\left(\frac{a}{2}+\frac{b}{p} r^{p-2}\right)\left\|v_{n}\right\|_{L_{Q}^{2}}^{2} \longrightarrow 0
\end{aligned}
$$


Now, for $n$ large enough, we have

$$
\int_{\mathbb{R}} e^{Q(t)}\left[\frac{1}{2} \nabla W\left(t, u_{n}\right) \cdot u_{n}-W\left(t, u_{n}\right)\right] d t=\psi\left(u_{n}\right)-\frac{1}{2} \psi^{\prime}\left(u_{n}\right) u_{n} \leq c+1
$$

which with Hölder's inequality and assumption $\left(W_{4}\right)$ implies

$$
\begin{aligned}
& \int_{\Lambda_{n}(r, \infty)} e^{Q(t)} \frac{W\left(t, u_{n}\right)}{\left|u_{n}\right|^{2}}\left|v_{n}\right|^{2} d t \\
& \leq\left(\int_{\Lambda_{n}(r, \infty)} e^{Q(t)}\left(\frac{W\left(t, u_{n}\right)}{\left|u_{n}\right|^{2}}\right)^{\sigma} d t\right)^{\frac{1}{\sigma}}\left(\int_{\Lambda_{n}(r, \infty)} e^{Q(t)}\left|v_{n}\right|^{2 \sigma^{\prime}} d t\right)^{\frac{1}{\sigma^{\prime}}} \\
& \leq\left(2 c_{0}\right)^{\frac{1}{\sigma}}\left(\int_{\Lambda_{n}(r, \infty)} e^{Q(t)}\left[\frac{1}{2} \nabla W\left(t, u_{n}\right) \cdot u_{n}-W\left(t, u_{n}\right)\right] d t\right)^{\frac{1}{\sigma}}\left(\int_{\Lambda_{n}(r, \infty)} e^{Q(t)}\left|v_{n}\right|^{2 \sigma^{\prime}} d t\right)^{\frac{1}{\sigma^{\prime}}} \\
& \leq\left(2 c_{0}(c+1)\right)^{\frac{1}{\sigma}}\left\|v_{n}\right\|_{L_{Q}^{2 \sigma^{\prime}}}^{2} \longrightarrow 0 .
\end{aligned}
$$

Combining (3.5) and (3.6) yields

$$
\int_{\mathbb{R}} e^{Q(t)} \frac{W\left(t, u_{n}\right)}{\left|u_{n}\right|^{2}}\left|v_{n}\right|^{2} d t=\int_{\Lambda_{n}(0, r)} e^{Q(t)} \frac{W\left(t, u_{n}\right)}{\left|u_{n}\right|^{2}}\left|v_{n}\right|^{2} d t+\int_{\Lambda_{n}(r, \infty)} e^{Q(t)} \frac{W\left(t, u_{n}\right)}{\left|u_{n}\right|^{2}}\left|v_{n}\right|^{2} d t \longrightarrow 0,
$$

which contradicts (3.4). Hence $\left(u_{n}\right)$ is bounded. Up to a subsequence, we can assume that $u_{n} \longrightarrow u$ in both $L_{Q}^{2}(\mathbb{R})$ and $L_{Q}^{p}(\mathbb{R})$. It follows from $\left(W_{1}\right)$ and Hölder's inequality that

$$
\begin{aligned}
\left|\int_{\mathbb{R}} e^{Q(t)} \nabla W\left(t, u_{n}\right) \cdot\left(u_{n}-u\right) d t\right| & \leq \int_{\mathbb{R}} e^{Q(t)}\left(a\left|u_{n}\right|+b\left|u_{n}\right|^{p-1}\right)\left|u_{n}-u\right| d t \\
& \leq a \int_{\mathbb{R}} e^{Q(t)}\left|u_{n}\right|\left|u_{n}-u\right| d t+b \int_{\mathbb{R}} e^{Q(t)}\left|u_{n}\right|^{p-1}\left|u_{n}-u\right| d t \\
& \leq\left\|u_{n}\right\|_{L_{Q}^{2}}\left\|u_{n}-u\right\|_{L_{Q}^{2}}+b\left\|u_{n}\right\|_{L_{Q}^{p}}^{p-1}\left\|u_{n}-u\right\|_{L_{Q}^{p}} \longrightarrow 0 .
\end{aligned}
$$

Therefore, we have

$$
\begin{aligned}
0 & =\lim _{n \longrightarrow \infty} \psi^{\prime}\left(u_{n}\right)\left(u_{n}-u\right) \\
& =\lim _{n \longrightarrow \infty}<u_{n}, u_{n}-u>-\lim _{n \longrightarrow \infty} \int_{\mathbb{R}} e^{Q(t)} \nabla W\left(t, u_{n}\right) \cdot\left(u_{n}-u\right) d t \\
& =\lim _{n \longrightarrow \infty}\left\|u_{n}\right\|^{2}-\|u\|^{2} .
\end{aligned}
$$

That is $\lim _{n \longrightarrow \infty}\left\|u_{n}\right\|^{2}=\|u\|^{2}$, which with $u_{n} \rightarrow u$ in $E$ implies

$$
\left\|u_{n}-u\right\|^{2}=<u_{n}-u, u_{n}-u>\longrightarrow 0 .
$$

Hence $\left(u_{n}\right)$ possesses a convergent subsequence in $E$. Thus $\psi$ satisfies the $(C)_{c}-$ condition. The proof of Lemma 3.3 is completed.

Consequently, Lemma 2.2 with Remark 2.1 imply that the functional $\psi$ possesses an unbounded sequence of critical points. Therefore, the damped vibration system $(\mathscr{D} \mathscr{V})$ possesses infinitely many fast homoclinic solutions. The proof is finished. 


\subsection{Proof of Theorem 3.2.}

Lemma 3.4. Assume that $\left(L_{1}^{\prime}\right),\left(L_{Q}\right),\left(W_{1}\right),\left(W_{2}\right)$ and $\left(W_{4}^{\prime}\right)$ are satisfied. Then for all positive constant $c$, $\psi$ satisfies the $(C)_{c}$-condition.

Proof. Let $c$ be a positive real and $\left(u_{n}\right) \subset E$ be a $(C)_{c}$-sequence, that is,

$$
\psi\left(u_{n}\right) \longrightarrow c \text { and }\left\|\psi^{\prime}\left(u_{n}\right)\right\|\left(1+\left\|u_{n}\right\|\right) \longrightarrow 0 \text { as } n \longrightarrow \infty .
$$

Assume by contradiction that $\left(u_{n}\right)$ is not bounded, then up to a subsequence, we can assume that $\left\|u_{n}\right\| \longrightarrow \infty$ as $n \longrightarrow \infty$. Letting $v_{n}=\frac{u_{n}}{\left\|u_{n}\right\|}$, one has $\left\|v_{n}\right\|=1$. By $\left(H_{4}^{\prime}\right)$, for $n$ large enough, one has

$$
\begin{aligned}
c+1 & \geq \psi\left(u_{n}\right)-\frac{1}{\mu} \psi^{\prime}\left(u_{n}\right) u_{n} \\
& =\frac{\mu-2}{2 \mu}\left\|u_{n}\right\|^{2}+\int_{\mathbb{R}} e^{Q(t)}\left[\frac{1}{\mu} \nabla W\left(t, u_{n}\right) \cdot u_{n}-W\left(t, u_{n}\right)\right] d t \\
& \geq \frac{\mu-2}{2 \mu}\left\|u_{n}\right\|^{2}-\frac{\gamma}{\mu}\left\|u_{n}\right\|_{2}^{2} .
\end{aligned}
$$

It follows that

$$
\limsup _{n \rightarrow \infty}\left\|v_{n}\right\|_{2}^{2} \geq \frac{\mu-2}{2 \gamma}
$$

Note $\left\|v_{n}\right\|=1$. Passing to a subsequence, $v_{n} \rightarrow v$ in $E$, we conclude from Lemma 2.1 that $v_{n} \longrightarrow v$ in $L_{Q}^{2}(\mathbb{R})$, which together with (3.7) implies that $v \neq 0$. Similar to (3.3), we get a contradiction. Therefore $\left(u_{n}\right)$ is bounded. The rest of the proof is the same as that in Lemma 3.3 and the proof of Lemma 3.4 is completed.

We conclude as in the proof of Theorem 3.1 that the functional $\psi$ possesses an unbounded sequence of critical points and the proof of Theorem 3.2 is finished.

\section{COMBINED NONLINEARITIES}

In this section, we consider the system $(\mathscr{D} \mathscr{V})$ involving a combination of superquadratic and subquadratic terms at infinty. More precisely, we take $W(t, x)=U(t, x)+V(t, x)$, where $U, V: \mathbb{R} \times \mathbb{R}^{N} \longrightarrow \mathbb{R}$ are continuous functions, differentiable with respect to the second variable with continuous derivatives $\nabla U(t, x)=\frac{\partial U}{\partial x}(t, x)$ and $\nabla V(t, x)=\frac{\partial V}{\partial x}(t, x)$. Consider the following conditions.

$\left(W_{5}\right) U(t, 0)=0$ and there exist constants $1<\gamma<2,1<\sigma<2$ and functions $c_{0}, a \in L_{Q}^{\frac{2}{2-\gamma}}\left(\mathbb{R}, \mathbb{R}^{+}\right)$ and $b \in L_{Q}^{\frac{2}{2-\sigma}}\left(\mathbb{R}, \mathbb{R}^{+}\right)$such that

$$
c_{0}(t)|x|^{\gamma} \leq \nabla U(t, x) \cdot x,|\nabla U(t, x)| \leq a(t)|x|^{\gamma-1}+b(t)|x|^{\sigma-1}, \text { a.e. } t \in \mathbb{R}, \forall x \in \mathbb{R}^{N} ;
$$

$\left(W_{6}\right) V(t, x) \geq 0$ for all $(t, x) \in \mathbb{R} \times \mathbb{R}^{N}$ and there exist $\mu>2, c \in L_{Q}^{2}\left(\mathbb{R}, \mathbb{R}^{+}\right)$and $d \in L^{\infty}\left(\mathbb{R}, \mathbb{R}^{+}\right)$ such that

$$
|\nabla V(t, x)| \leq c(t)+d(t)|x|^{\mu-1} \text {, a.e. } t \in \mathbb{R}, \forall x \in \mathbb{R}^{N} ;
$$

$\left(W_{7}\right)$ There exist $\rho>2,1<\delta<2$ and $\theta \in C\left(\mathbb{R}, \mathbb{R}^{+}\right) \cap L_{Q}^{\frac{2}{2-\delta}}\left(\mathbb{R}, \mathbb{R}^{+}\right)$such that

$$
\rho V(t, x)-\nabla V(t, x) \cdot x \leq \theta(t)|x|^{\delta} \text {, a.e. } t \in \mathbb{R}, \forall x \in \mathbb{R}^{N} .
$$

Now, we state the main result of this section. 
Theorem 4.1. Assume that $\left(L_{1}^{\prime}\right),\left(L_{Q}\right),\left(W_{3}\right)$ and $\left(W_{5}\right)-\left(W_{7}\right)$ are satisfied. Then system $(\mathscr{D} \mathscr{V})$ possesses infinitely many fast homoclinic solutions.

Example 4.1. Let $W(t, x)=U(t, x)+V(t, x)$, where

$$
U(t, x)=\left(\frac{1}{1+|t|^{2}}\right)^{\frac{1}{3}}|x|^{\frac{4}{3}}+\left(\frac{1}{1+|t|^{2}}\right)^{\frac{1}{6}}|x|^{\frac{5}{3}},
$$

and

$$
V(t, x)=\left(\frac{1}{1+|t|^{2}}\right)\left[|x|^{\frac{4}{3}} \ln (1+|x|)+|x|^{3}\right]
$$

By a classical computation, we check that $\left(W_{3}\right),\left(W_{5}\right)-\left(W_{7}\right)$ are satisfied. Hence the corresponding system $(\mathscr{D} \mathscr{V})$ possesses infinitely many fast homoclinic solutions. Let us remark that $U(t, x)$ is subquadratic and $V(t, x)$ is superquadratic but do not satisfy the $(\mathscr{A} \mathscr{R})$ condition.

Consider the variational functional $\psi$ associated to $(\mathscr{D} \mathscr{V})$ :

$$
\psi(u)=\frac{1}{2} \int_{\mathbb{R}} e^{Q(t)}\left[|\dot{u}(t)|^{2}+L(t) u(t) \cdot u(t)\right] d t-\int_{\mathbb{R}} e^{Q(t)} W(t, u) d t
$$

defined in Section 3.

Lemma 4.1. Assume that $\left(L_{1}^{\prime}\right),\left(L_{Q}\right),\left(W_{5}\right)$ and $\left(W_{6}\right)$ are satisfied. If $u_{n} \rightarrow u$ in $E$, then

$$
\nabla W\left(., u_{n}\right) \longrightarrow \nabla W(., u) \in L_{Q}^{2}(\mathbb{R}) .
$$

Proof. Arguing indirectly, by Lemma 2.1, there exists a subsequence $\left(u_{n_{p}}\right)$ such that

$$
u_{n_{p}} \longrightarrow \text { u in both } L_{Q}^{2}(\mathbb{R}) \text { and } L_{Q}^{2(\mu-1)}(\mathbb{R}) \text { and } u_{n_{p}} \longrightarrow \text { u a.e. in } \mathbb{R} \text { as } p \longrightarrow \infty
$$

and

$$
\int_{\mathbb{R}} e^{Q(t)}\left|\nabla W\left(t, u_{n_{p}}(t)\right)-\nabla W(t, u(t))\right|^{2} d t \geq \varepsilon_{0}, \forall p \in \mathbb{N}
$$

for some positive constant $\varepsilon_{0}$. By (4.2) and up to a subsequence if necessary, we can assume that

$$
\sum_{p=1}^{\infty}\left\|u_{n_{p}}-u\right\|_{L_{Q}^{2}}<\infty
$$

and

$$
\sum_{p=1}^{\infty}\left\|u_{n_{p}}-u\right\|_{L_{Q}^{2(\mu-1)}}<\infty
$$


Let $w(t)=\sum_{p=1}^{\infty}\left|u_{n_{p}}(t)-u(t)\right|$ for all $t \in \mathbb{R}$. Then $w \in L_{Q}^{2}(\mathbb{R}) \cap L_{Q}^{2(\mu-1)}(\mathbb{R})$. By $\left(W_{5}\right)$ and $\left(W_{6}\right)$, there holds for all $p \in \mathbb{N}$ and $t \in \mathbb{R}$

$$
\begin{aligned}
& \left|\nabla W\left(t, u_{n_{p}}\right)-\nabla W(t, u)\right|^{2} \\
& \leq\left[\left|\nabla W\left(t, u_{n_{p}}\right)\right|+|\nabla W(t, u)|\right]^{2} \\
& \leq\left[\left|\nabla U\left(t, u_{n_{p}}\right)\right|+\left|\nabla V\left(t, u_{n_{p}}\right)\right|+|\nabla U(t, u)|+|\nabla V(t, u)|\right]^{2} \\
& \leq\left[a\left|u_{n_{p}}\right|^{\gamma-1}+b\left|u_{n_{p}}\right|^{\sigma-1}+a|u|^{\gamma-1}+b|u|^{\sigma-1}\right. \\
& \left.+2 c+d\left|u_{n_{p}}\right|^{\mu-1}+d|u|^{\mu-1}\right]^{2} \\
& \leq\left[a\left(\left|u_{n_{p}}-u\right|+|u|\right)^{\gamma-1}+b\left(\left|u_{n_{p}}-u\right|+|u|\right)^{\sigma-1}+a|u|^{\gamma-1}+b|u|^{\sigma-1}\right. \\
& \left.+2 c+d\left(\left|u_{n_{p}}-u\right|+|u|\right)^{\mu-1}+d|u|^{\mu-1}\right]^{2} \\
& \leq\left[a(w+|u|)^{\gamma-1}+b(w+|u|)^{\sigma-1}+a|u|^{\gamma-1}+b|u|^{\sigma-1}\right. \\
& \left.+2 c+d(w+|u|)^{\mu-1}+d|u|^{\mu-1}\right]^{2} \\
& \leq c_{1}\left[a^{2} w^{2(\gamma-1)}+a^{2}|u|^{2(\gamma-1)}+b^{2} w^{2(\sigma-1)}+b^{2}|u|^{2(\sigma-1)}\right. \\
& \left.+c^{2}+d^{2} w^{2(\mu-1)}+d^{2}|u|^{2(\mu-1)}\right]=k(t)
\end{aligned}
$$

where $c_{1}$ is a positive constant. It is easy to see that $k \in L_{Q}^{1}(\mathbb{R})$. Hence, combining (4.2) with (4.4), Lebesgue's Dominated Convergence Theorem implies

$$
\lim _{p \longrightarrow \infty} \int_{\mathbb{R}} e^{Q(t)}\left|\nabla W\left(t, u_{n_{p}}(t)\right)-\nabla W(t, u(t))\right|^{2} d t=0,
$$

which contradicts with (4.3). Hence (4.1) is true. The proof of Lemma 4.1 is completed.

Under the assumptions of Theorem 4.1, it is well known that $\psi \in C^{1}(E, \mathbb{R})$ and for all $u, v \in E$

$$
\begin{aligned}
\psi^{\prime}(u) v & =\int_{\mathbb{R}} e^{Q(t)}[\dot{u}(t) \cdot \dot{v}(t)+L(t) u(t) \cdot v(t)-\nabla W(t, u) \cdot v] d t \\
& =<u, v>-\int_{\mathbb{R}} e^{Q(t)} \nabla W(t, u) \cdot v d t .
\end{aligned}
$$

Moreover, $\psi^{\prime}: E \longrightarrow E^{*}$ is compact and any critical point of $\psi$ on $E$ is a fast homoclinic solution for system $(\mathscr{D} \mathscr{V})$.

In the next, we prove Theorem 4.1 by applying Lemma 2.3. Choose a completely orthonormal basis $\left(e_{j}\right)$ of $E$ and define $X_{j}=\mathbb{R} e_{j}$. Then, $Y_{k}$ and $Z_{k}$ can be defined as in Section 2. By $\left(W_{3}\right)$, one has that $\psi$ is even. In the following, we will check that all the conditions of Lemma 2.3 are satisfied.

Lemma 4.2. Assume that $\left(L_{1}^{\prime}\right),\left(L_{Q}\right),\left(W_{5}\right)$ and $\left(W_{7}\right)$ are satisfied. Then $\psi$ satisfies the $(P S)^{*}$ condition. 
Proof. Let $\left(u_{j}\right)$ be a $(P S)^{*}$-sequence, that is, $\left(\psi\left(u_{j}\right)\right)$ is bounded, $u_{j} \in Y_{k_{j}}$ for some $k_{j}$ with $k_{j} \longrightarrow \infty$ and $\left(\psi_{\mid Y_{k_{j}}}\right)^{\prime}\left(u_{j}\right) \longrightarrow 0$ as $j \longrightarrow \infty$. Now, we show that $\left(u_{j}\right)$ is bounded in $E$. By virtue of the Mean Value Theorem, $(2.1),\left(W_{5}\right)$ and $\left(W_{7}\right)$, there exists a positive constant $M$ such that

$$
\begin{aligned}
\rho M+M\left\|u_{j}\right\| & \geq \rho \psi\left(u_{j}\right)-\psi^{\prime}\left(u_{j}\right) u_{j} \\
& =\left(\frac{\rho}{2}-1\right)\left\|u_{j}\right\|^{2}+\int_{\mathbb{R}} e^{Q(t)}\left[\nabla W\left(t, u_{j}\right) \cdot u_{j}-\rho W\left(t, u_{j}\right)\right] d t \\
& =\left(\frac{\rho}{2}-1\right)\left\|u_{j}\right\|^{2}+\int_{\mathbb{R}} e^{Q(t)}\left[\nabla U\left(t, u_{j}\right) \cdot u_{j}-\rho U\left(t, u_{j}\right)\right] d t \\
& +\int_{\mathbb{R}} e^{Q(t)}\left[\nabla V\left(t, u_{j}\right) \cdot u_{j}-\rho V\left(t, u_{j}\right)\right] d t \\
& \geq\left(\frac{\rho}{2}-1\right)\left\|u_{j}\right\|^{2}-\int_{\mathbb{R}} e^{Q(t)}\left[a(t)\left|u_{j}\right|^{\gamma}+b(t)\left|u_{j}\right|^{\sigma}\right] d t \\
& -\rho \int_{\mathbb{R}} e^{Q(t)}\left[\frac{a(t)}{\gamma}\left|u_{j}\right|^{\gamma}+\frac{b(t)}{\sigma}\left|u_{j}\right|^{\sigma}\right] d t-\int_{\mathbb{R}} e^{Q(t)} \theta(t)\left|u_{j}\right|^{\delta} d t \\
& \geq\left(\frac{\rho}{2}-1\right)\left\|u_{j}\right\|^{2}-\left(1+\frac{\rho}{\gamma}\right)\|a\|_{L_{Q}^{2-\gamma}}^{2-\gamma}\left\|u_{j}\right\|_{L_{Q}^{2}}^{\gamma} \\
& -\left(1+\frac{\rho}{\sigma}\right)\|b\|_{L_{Q}^{2-\sigma}}^{\frac{2}{2-\sigma}}\left\|u_{j}\right\|_{L_{Q}^{2}}^{\sigma}-\|\theta\|_{L_{Q}^{2-\delta}}^{\frac{2}{2-\delta}}\left\|u_{j}\right\|_{L_{Q}^{2}}^{\delta} \\
& \geq\left(\frac{\rho}{2}-1\right)\left\|u_{j}\right\|^{2}-\left(1+\frac{\rho}{\gamma}\right) \eta_{2}^{\gamma}\|a\|_{L_{Q}^{2-\gamma}}\left\|u_{j}\right\|^{\gamma} \\
& -\left(1+\frac{\rho}{\sigma}\right) \eta_{2}^{\sigma}\|b\|_{L_{Q}^{2-\sigma}}^{2}\left\|u_{j}\right\|^{\sigma}-\|\theta\|_{L_{Q}^{2-\delta}} \eta_{2}^{\delta}\left\|u_{j}\right\|^{\delta} .
\end{aligned}
$$

Since $\rho>2$ and $\gamma, \sigma, \delta<2$, it follows that $\left(u_{j}\right)$ is bounded in $E$. From the reflexivity of $E$ and up to a subsequence if necessary, we may assume that $u_{j} \rightarrow u$ in $E$, for some $u \in E$. Now, we have

$$
\left\|u_{j}-u\right\|^{2}=\left(\psi^{\prime}\left(u_{j}\right)-\psi^{\prime}(u)\right)\left(u_{j}-u\right)+\int_{\mathbb{R}} e^{Q(t)}\left(\nabla W\left(t, u_{j}\right)-\nabla W(t, u)\right) \cdot\left(u_{j}-u\right) d t .
$$

It is clear that $\left(\psi^{\prime}\left(u_{j}\right)-\psi^{\prime}(u)\right)\left(u_{j}-u\right) \longrightarrow 0$ as $j \longrightarrow \infty$. By Hölder's inequality, (2.1) and Lemma 4.1, one has

$$
\begin{aligned}
& \left|\int_{\mathbb{R}} e^{Q(t)}\left(\nabla W\left(t, u_{j}\right)-\nabla W(t, u)\right) \cdot\left(u_{j}-u\right) d t\right| \\
& \leq\left\|\nabla W\left(., u_{j}\right)-\nabla W(., u)\right\|_{L_{Q}^{2}}\left\|u_{j}-u\right\|_{L_{Q}^{2}} \\
& \leq \eta_{2}\left\|\nabla W\left(., u_{j}\right)-\nabla W(., u)\right\|_{L_{Q}^{2}}\left\|u_{j}-u\right\| \longrightarrow 0 \text { as } j \longrightarrow \infty .
\end{aligned}
$$

Combining (4.5)-(4.6), we deduce that $u_{j} \longrightarrow u$ in $E$ and the proof of Lemma 4.2 is completed.

Lemma 4.3. Assume that $\left(L_{1}^{\prime}\right),\left(L_{Q}\right),\left(W_{5}\right)$ and $\left(W_{6}\right)$ are satisfied. Then for any sufficiently large $k \in \mathbb{N}$, there exists a constant $\rho_{k}>0$ such that $a_{k}=\inf _{u \in Z_{k},\|u\|=\rho_{k}} \psi(u) \geq 0$. 
Proof. Let $l_{2}(k)$ be defined in the proof of Lemma 3.1. By the Mean Value Theorem, $\left(H_{5}\right),\left(H_{6}\right)$ and (2.1), we have, for any $u \in Z_{k}$,

$$
\begin{aligned}
\psi(u) & =\frac{1}{2}\|u\|^{2}-\int_{\mathbb{R}} e^{Q(t)} W(t, u) d t \\
& \geq \frac{1}{2}\|u\|^{2}-\int_{\mathbb{R}} e^{Q(t)}\left[\frac{1}{\gamma} a|u|^{\gamma}+\frac{1}{\sigma} b|u|^{\sigma}\right] d t-\int_{\mathbb{R}} e^{Q(t)}\left[c|u|+\frac{1}{\mu} d|u|^{\mu}\right] d t \\
& \geq \frac{1}{2}\|u\|^{2}-\frac{1}{\gamma}\|a\|_{L_{Q}^{2-\gamma}}\|u\|_{L_{Q}^{2}}^{\gamma}-\frac{1}{\sigma}\|b\|_{L_{Q}^{\frac{2}{2-\sigma}}\|u\|_{L_{Q}^{2}}^{\sigma}} \\
& -\|c\|_{L_{Q}^{2}}\|u\|_{L_{Q}^{2}}-\frac{1}{\mu}\|d\|_{L^{\infty}}\|u\|_{L_{Q}^{\mu}}^{\mu} \\
& \geq \frac{1}{2}\|u\|^{2}-\frac{1}{\gamma} l_{2}^{\gamma}(k)\|a\|_{L_{Q}^{2-\gamma}}\|u\|^{\gamma}-\frac{1}{\sigma} l_{2}^{\sigma}(k)\|b\|_{L_{Q}^{2-\sigma}}\|u\|^{\sigma} \\
& -l_{2}(k)\|c\|_{L_{Q}^{2}}\|u\|-\frac{1}{\mu} \eta_{\mu}^{\mu}\|d\|_{L^{\infty}}\|u\|^{\mu} .
\end{aligned}
$$

In view of (4.7), $\mu>2$ and $\gamma, \sigma>1$, one has

$$
\psi(u) \geq \frac{1}{4}\|u\|^{2}-\left(\frac{1}{\gamma} l_{2}^{\gamma}(k)\|a\|_{L_{Q}^{2-\gamma}}+\frac{1}{\sigma} l_{2}^{\sigma}(k)\|b\|_{L_{Q}^{2-\sigma}}+l_{2}(k)\|c\|_{L_{Q}^{2}}\right)\|u\|
$$

for $\|u\| \leq \inf \left\{1,\left(\frac{\mu}{4\|d\|_{L^{\infty}} \eta_{\mu}^{\mu}}\right)^{\frac{1}{\mu-2}}\right\}$. Let

$$
\rho_{k}=8\left(\frac{1}{\gamma} l_{2}^{\gamma}(k)\|a\|_{L_{Q}^{2-\gamma}}+\frac{1}{\sigma} l_{2}^{\sigma}(k)\|b\|_{L_{Q}^{\frac{2}{2-\sigma}}}+l_{2}(k)\|c\|_{L_{Q}^{2}}\right) .
$$

It is easy to see that $\rho_{k} \longrightarrow 0$ as $k \longrightarrow \infty$. Thus, for sufficiently large integer $k,(4.8)$ implies $a_{k} \geq \frac{1}{8} \rho_{k}^{2}>0$. The proof of Lemma 4.3 is completed.

Lemma 4.4. Assume that $\left(L_{1}^{\prime}\right),\left(L_{Q}\right),\left(W_{5}\right)$ and $\left(W_{6}\right)$ are satisfied. Then $d_{k}=\inf _{u \in Z_{k},\|u\| \leq \rho_{k}} \psi(u)$ $\longrightarrow 0$ as $k \longrightarrow \infty$.

Proof. By (4.8), for any $u \in Z_{k}$, we have

$$
\psi(u) \geq-\left(\frac{1}{\gamma} l_{2}^{\gamma}(k)\|a\|_{L_{Q}^{2-\gamma}}+\frac{1}{\sigma} l_{2}^{\sigma}(k)\|b\|_{L_{Q}^{\frac{2}{2-\sigma}}}+l_{2}(k)\|c\|_{L_{Q}^{2}}\right)\|u\| .
$$

Therefore, we get with $\|u\| \leq \rho_{k}$

$$
0 \geq d_{k} \geq-\left(\frac{1}{\gamma} l_{2}^{\gamma}(k)\|a\|_{L_{Q}^{2-\gamma}}+\frac{1}{\sigma} l_{2}^{\sigma}(k)\|b\|_{L_{Q} \frac{2}{2-\sigma}}+l_{2}(k)\|c\|_{L_{Q}^{2}}\right) \rho_{k} .
$$

Since $l_{2}(k), \rho_{k} \longrightarrow 0$ as $k \longrightarrow \infty$, one has $d_{k} \longrightarrow 0$ as $k \longrightarrow \infty$. The proof of Lemma 4.4 is completed.

Lemma 4.5. Assume that $\left(L_{1}^{\prime}\right),\left(L_{Q}\right),\left(W_{5}\right)$ and $\left(W_{6}\right)$ are satisfied. Then $b_{k}=\inf _{u \in Y_{k},\|u\|=r_{k}} \psi(u)<$ $0, \forall k \in \mathbb{N}$. 
Proof. First, we claim that there exists $\varepsilon>0$ such that

$$
\operatorname{meas}_{Q}\left(\left\{t \in \mathbb{R} / c_{0}(t)|u(t)|^{\gamma} \geq \varepsilon\|u\|^{\gamma}\right\}\right) \geq \varepsilon, \forall u \in Y_{k} \backslash\{0\} .
$$

If not, there exists a sequence $\left(u_{n}\right) \subset Y_{k}$ with $\left\|u_{n}\right\|=1$ such that

$$
\operatorname{meas}_{Q}\left(\left\{t \in \mathbb{R} / c_{0}(t)\left|u_{n}(t)\right|^{\gamma} \geq \frac{1}{n}\right\}\right) \leq \frac{1}{n} .
$$

Since $\operatorname{dim} Y_{k}<\infty$, it follows from the compactness of the unit sphere of $Y_{k}$ that there exists a subsequence, say $\left(u_{n}\right)$, such that $\left(u_{n}\right)$ converges to some $u \in Y_{k}$. Hence, $\|u\|=1$. Since all norms are equivalent in the finite-dimensional space $Y_{k}$, we have $u_{n} \longrightarrow u$ in $L_{Q}^{2}(\mathbb{R})$. By the Hölder's inequality, one has

$$
\int_{\mathbb{R}} e^{Q(t)} c_{0}(t)\left|u_{n}-u\right|^{\gamma} d t \leq\left\|c_{0}\right\|_{L_{Q}^{2-\gamma}}\left\|u_{n}-u\right\|_{L_{Q}^{2}}^{\mu} \longrightarrow 0 \text { as } n \longrightarrow \infty .
$$

Thus, there exists $\varepsilon_{0}>0$ such that

$$
\operatorname{meas}_{Q}\left(\left\{t \in \mathbb{R} / c_{0}(t)|u(t)|^{\gamma} \geq \varepsilon_{0}\right\}\right) \geq \varepsilon_{0} .
$$

In fact, if not, we have, for all $n \in \mathbb{N}$,

$$
\operatorname{meas}_{Q}\left(\left\{t \in \mathbb{R} / c_{0}(t)|u(t)|^{\gamma} \geq \frac{1}{n}\right\}\right) \leq \frac{1}{n} .
$$

Let $n \in \mathbb{N}$, then, for all integer $m \geq n$,

$$
\operatorname{meas}_{Q}\left(\left\{t \in \mathbb{R} / c_{0}(t)|u(t)|^{\gamma} \geq \frac{1}{n}\right\}\right) \leq \operatorname{meas}_{Q}\left(\left\{t \in \mathbb{R} / c_{0}(t)|u(t)|^{\gamma} \geq \frac{1}{m}\right\}\right) \leq \frac{1}{m}
$$

which implies

$$
\operatorname{meas}_{Q}\left(\left\{t \in \mathbb{R} / c_{0}(t)|u(t)|^{\gamma} \geq \frac{1}{n}\right\}\right)=0
$$

So

$$
\begin{aligned}
\int_{\mathbb{R}} e^{Q(t)} c_{0}(t)|u|^{\gamma+2} d t & =\int_{\left\{t \in \mathbb{R} / c_{0}(t)|u(t)|^{\gamma} \leq \frac{1}{n}\right\}} e^{Q(t)} c_{0}(t)|u|^{\gamma+2} d t \\
& \leq \frac{1}{n} \int_{\mathbb{R}} e^{Q(t)}|u|^{2} d t \\
& \leq \frac{\eta_{2}^{2}}{n}\|u\|^{2}=\frac{\eta_{2}^{2}}{n} \longrightarrow 0 \text { as } n \longrightarrow \infty
\end{aligned}
$$

Hence $u=0$, which contradicts $\|u\|=1$. Therefore (4.12) holds. Thus, define

$$
\Omega_{0}=\left\{t \in \mathbb{R} / c_{0}(t)|u(t)|^{\gamma} \geq \varepsilon_{0}\right\}, \Omega_{n}=\left\{t \in \mathbb{R} / c_{0}(t)\left|u_{n}(t)\right|^{\gamma} \leq \frac{1}{n}\right\} .
$$

Combining (4.10) and (4.12), we obtain

$$
\begin{aligned}
\operatorname{meas}_{Q}\left(\Omega_{0} \bigcap \Omega_{n}\right) & =\text { meas }_{Q}\left(\Omega_{0} \backslash\left(\Omega_{n}^{c} \bigcap \Omega_{0}\right)\right) \\
& \geq \operatorname{meas}_{Q}\left(\Omega_{0}\right)-\text { meas }_{Q}\left(\Omega_{n}^{c} \bigcap \Omega_{0}\right) \\
& \geq \varepsilon_{0}-\frac{1}{n}, \forall n \in \mathbb{N} .
\end{aligned}
$$


Let $n$ be large enough such that $\varepsilon_{0}-\frac{1}{n} \geq \frac{1}{2} \varepsilon_{0}$ and $\frac{\varepsilon_{0}}{2^{\gamma-1}}-\frac{1}{n} \geq \frac{\varepsilon_{0}}{2^{\gamma}}$. Then,

$$
\begin{aligned}
\int_{\mathbb{R}} e^{Q(t)} c_{0}(t)\left|u_{n}-u\right|^{\gamma} d t & \geq \int_{\Omega_{0} \cap \Omega_{n}} e^{Q(t)} c_{0}(t)\left|u_{n}-u\right|^{\gamma} d t \\
& \geq\left(\frac{\varepsilon_{0}}{2^{\gamma-1}}-\frac{1}{n}\right) \operatorname{meas}_{Q}\left(\Omega_{0} \bigcap \Omega_{n}\right) \\
& \geq \frac{\varepsilon_{0}^{2}}{2^{\gamma+1}}
\end{aligned}
$$

for all large integer $n$, which is a contradiction to (4.11). Therefore (4.9) holds. For the $\varepsilon$ given in (4.9), let

$$
\left.\Omega_{u}=\left\{t \in \mathbb{R} / c_{0}(t)|u(t)|^{\gamma} \geq \varepsilon\|u\|^{\gamma}\right\}\right), \forall u \in Y_{k} \backslash\{0\} .
$$

By (4.9), we obtain

$$
\operatorname{meas}_{Q}\left(\Omega_{u}\right) \geq \varepsilon, \forall u \in Y_{k} \backslash\{0\} .
$$

For any $u \in Y_{k}$, by the Mean Value Theorem, $\left(W_{5}\right),\left(W_{6}\right),(4.13)$ and (4.14), one has

$$
\begin{aligned}
\psi(u) & =\frac{1}{2}\|u\|^{2}-\int_{\mathbb{R}} e^{Q(t)} W(t, u) d t \\
& \leq \frac{1}{2}\|u\|^{2}-\frac{1}{\gamma} \int_{\mathbb{R}} e^{Q(t)} c_{0}(t)|u|^{\gamma} d t-\int_{\mathbb{R}} e^{Q(t)} V(t, u) d t \\
& \leq \frac{1}{2}\|u\|^{2}-\frac{1}{\gamma} \int_{\Omega_{u}} c_{0}(t)|u|^{\gamma} d t \\
& \leq \frac{1}{2}\|u\|^{2}-\frac{\varepsilon}{\gamma}\|u\|^{\gamma} \operatorname{meas}_{Q}\left(\Omega_{u}\right) \\
& \leq \frac{1}{2}\|u\|^{2}-\frac{\varepsilon^{2}}{\gamma}\|u\|^{\gamma} .
\end{aligned}
$$

Choose $0<r_{k}<\inf \left\{\rho_{k},\left(\frac{\varepsilon^{2}}{\gamma}\right)^{\frac{1}{2-\gamma}}\right\}$. Direct computation shows that

$$
b_{k}=\inf _{u \in Y_{k},\|u\|=r_{k}} \psi(u) \leq \frac{1}{2} r_{k}^{2}-r_{k}^{2-\gamma} r_{k}^{\gamma}=-\frac{1}{2} r_{k}^{2}<0 .
$$

The proof of Lemma 4.5 is completed.

Therefore, Lemmas 4.2-4.5 imply that all the conditions of Lemma 2.3 are satisfied. Thus, it follows from Lemma 2.3 that $\psi$ has infinitely many nontrivial critical points, that is, equation $(\mathscr{D} \mathscr{V})$ possesses infinitely many fast homoclinic solutions.

Remark 4.1. In the case where the nonlinearity $W$ of the damped vibration system $(\mathscr{D} \mathscr{V})$ is superquadratic or involves a combination of superquadratic and subquadratic terms, we obtain the existence of infinitely many nontrivial fast homoclinic solutions for system $(\mathscr{D} \mathscr{V})$ by using variational methods and the critical point theory, which unify and sharply improve some recent results in the literature. To the best of our knowledge, there is no result available concerning these cases. Here we can give some nontrivial examples, which show that these assumptions of the nonlinearity $W$ are reasonable.

\section{Acknowledgments}


The author expresses his gratitude to the anonymous referees and the editor for their valuable comments and suggestions which have led to a significant improvement on the presentation and qualify of this paper.

\section{REFERENCES}

[1] M. Izydorek, J. Janczewska, Homoclinic solutions for a class of the second order Hamiltonian sysstems, J. Differential Equations 219 (2005), 375-389.

[2] J. Jiang, S. Lu, X. Lv, Homoclinic solutions for a class of second order Hamiltonian systems, Nonlinear Anal. Real word Appl. 13 (2012), 176-185.

[3] S. Lu, X. Lv, P. Yan, Existence of homoclinics for a class of Hamiltonian systems, Nonlinear Anal. 72 (2010), 390-398.

[4] P.H. Rabinowitz, Homoclinic orbits for a class of Hamiltonian systems, Proc. Roy. Soc. Edinburgh 114 (1990), 33-38.

[5] X.H. Tang, L. Xiao, Homoclinic solutionsd for a class of second order Hamiltonian systems, Nonlinear Anal. 71 (2009), 1140-1152.

[6] R. Yuan, Z. Zhang, Homoclinic solutions for some second order nonautonomous Hamiltonian systems without the globally superquadratic condition, Nonlinear Anal. 72 (2010), 1809-1819.

[7] P.H. Rabinowitz, K. Tanaka, Some results on connecting orbits for a class of Hamiltonian systems, Math. Z. 206 (1991), 473-499.

[8] G. Chen, Homoclinic orbits for second order Hamiltonian systems with asymptotically linear terms at infinity, Adv. Difference Equ. 2014 (2014), 114.

[9] G. Chen, Z. He, Infinitely many homoclinic solutions for a class of second order Hamiltonian systems, Adv. Difference Equ. 2014 (2014), 161.

[10] L. Chu, Q. Zhang, Homoclinic solutions for a class of second order Hamiltonian systems with locally defined potentials, Nonlinear Anal. 75 (2012), 3188-3197.

[11] X. Lin, X.H. Tang, Homoclinic solutions for a class of second order Hamiltonian systems, J. Math. Anal. Appl. 354 (2009), 539-549.

[12] X. Lin, X.H. Tang, Infinitely many homoclinic orbits for Hamiltonian systems with indefinite sign subquadratic potentials, Nonlinear Anal. 74 (2011), 6314-6325.

[13] X. Lin, X.H. Tang, New conditions on homoclinic solutions for a subquadratic second order Hamiltonian system, Boundary Value Probl. 2015 (2015), 111.

[14] C. Liu, Q. Zhang, Infinitely many homoclinic solutions for second order Hamiltonian systems, Nonlinear Anal. 72 (2010), 894-903.

[15] J. Sun, T-F. Wu, Homoclinic solutions for a second order Hamiltonian system with a positive semi-definite matrix Chaos, Solitons Fractals 76 (2015), 24-31.

[16] C.L. Tang, L.L. Wan, Existence of homoclinic orbits for second order Hamiltonian systems without (AR) condition, Nonlinear Anal. 74 (2011), 5303-5313.

[17] L.L. Wan, Existence and multiplicity of homoclinic orbits for second order Hamiltonian systems without (AR) condition, Discrete Continuous Dyn. sys. Serie B, 15 (2011), 255-271.

[18] J. Wei, J. Wang, Infinitely many homoclinic orbits for the second order Hamiltonian systems with general potentials, J. Math. Anal. Appl. 366 (2010), 694-699.

[19] Z. Zhang, Existence of homoclinic solutions for second order Hamiltonian systems with general potentials, J. Appl. Math. Comput. 44 (2014), 263-272.

[20] W. Jiang, Q. Zhang, Multiple homoclinic solutions for superquadratic Hamiltonian systems, Electron. J. Diff. Equ. 2016 (2016), 66.

[21] Y. Ding, Existence and multiplicity results for homoclinic solutions to a class of Hamiltonian systems, Nonlinear Anal. 25, (1995), 1095 -1113.

[22] J. Sun, T-f. Wu, Multiplicity and concentration of homoclinic solutions for some second order Hamiltonian systems, Nonlinear Anal. 114 (2015), 105-115.

[23] R.P. Agarwal, P. Chen, X. Tang, Fast homoclinic solutions for a class of damped vibration problems, Appl. Math. Comput. 219 (2013), 6053-6065. 
[24] P. Chen, X.H. Tang, Fast homoclinic solutions for a class of damped vibration problems with sub-quadratic potentials, Math. Nachr. 286 (2013), 4-16.

[25] F. Khelifi, M. Timoumi, Even homoclinic orbits for a class of damped vibration systems, Indigationes Math. 28 (2017), 1111-1125.

[26] Y. Li, Q. Zhang, Existence and multiplicity of fast homoclinic solutions for a class of nonlinear second order nonautonomous systems in a weighted Sobolev space, J. Funct. Spaces 2015 (2015), Article ID 495040.

[27] X. Wu, W. Zhang, Existence and multiplicity of homoclinic solutions for a class of damped vibration problems, Nonlinear Anal. 74 (2011), 4392-4398.

[28] R. Yuan, Z. Zhang, Fast homoclinic solutions for some second order non-autonomous systems, J. Math. Anal. Appl. 376 (2011), 51-63.

[29] M. Timoumi, Existence and multiplicity of fast homoclinic solutions for a class of damped vibration problems, J. Nonlinear Funct. Anal. 2016 (2016), Article ID 9.

[30] M. Timoumi, Ground state homoclinic orbits of a class of superquadratic damped vibration problems, Commun. Optim. Theory 2017 (2017), Article ID 29.

[31] P.H. Rabinowitz, Minimax methods in critical point theory with applications to differential equations; CBMS Reg. Conf. Ser. in Math., Vol. 65, American Mathematical Society, Providence, RI, 1986.

[32] T. Bartolo, V. Benci, D. Fortunato, Abstract critical point theorems and applications to some nonlinear problems with strong resonance at infinity, Nonlinear Anal. 7 (1983), 981-1012.

[33] M. Yang, Z. Han, Infinitely many homoclinic solutions for second order Hamiltonian systems with odd nonlinearities, Nonlinear Anal. 74 (2011), 2635-2646. 